\title{
ON THE DÏMENSION OF MODULES AND ALGEBRAS, VII ALGEBRAS WITH FINITE-DIMENSIONAL RESIDUE-ALGEBRAS
}

\author{
J. P. JANS and TADASI NAKAYAMA
}

It was shown in Eilenberg-Nagao-Nakayama [3] (Theorem 8 and §4) that if $\Omega$ is an algebra (with unit element) over a field $K$ with $(\Omega: K)<\infty$ and if the cohomolgical dimension of $\Omega, \operatorname{dim} \Omega$, is $\leqq 1$, then every residue-algebra of $\Omega$ has a finite cohomological dimension. In the present note we prove a theorem of converse type, which gives, when combined with the cited result, a rather complete general picture of algebras whose residue-algebras are all of finite cohomological dimension. Namely, if $\Lambda$ is an algebra over a field $K$ with $(\Lambda: K)<\infty$ and if

$$
\operatorname{dim}\left(\Lambda / N^{2}\right)<\infty,
$$

where $N$ is the radical of $\Lambda$, then $\Lambda$ is a homomorphic image of an algebra $\Omega$ over $K$ with $(\Omega: K)<\infty$ such that

$$
\operatorname{dim} \Omega \leqq 1 .
$$

We may further impose the condition

$$
\Omega / M^{2} \approx 1 / N^{2}
$$

where $M$ is the radical of $\Omega$, and with this additional condition the algebra $\Omega$ and the homomorphism $\Omega \rightarrow A$ are determined uniquely up to an isomorphism.

Thus, algebras with cohomological dimension $\leqq 1$ are in a sense "prototypes" for algebras with finite-dimensional residue-algebras. The construction of $\Omega$ and the homomorphism $\Omega \rightarrow A$ is essentially what was employed by Hochschild $[5,6]$ in connection with his notion of "maximal algebra" and by Jans [3] as free algebras.

We shall start with semi-primary rings (in the sense in [3]). For them and for their global dimensions we shall prove a theorem which is quite similar as above but which assumes an additional condition on "splitting".

Received April 14, 1956. 


\section{§ 1. Rings with $N^{2}=0$}

In this section $A$ will denote a semi-primary ring with radical $N$ such that $N^{2}=0$. The quotient ring $\Gamma=\Lambda / N$ is then semi-simple and $N$ is a two-sided $\Gamma$-module.

LemMa 1. Let $e, e^{\prime}$ be primitive idempotents in $\Gamma$ such that

$$
N e \neq 0 \neq e N e^{\prime}
$$

Then

$$
0 \leqq 1 \cdot \operatorname{dim}_{\Delta} N e<1 . \operatorname{dim}_{\Delta} N e^{\prime} .
$$

Proof. Our lemma (as well as Proposition 2 below) follows readily from the consideration of "minimal resolution" (i.e. a projective resolution consisting of "minimal homomorphisms") (Eilenberg-Nakayama [4], Eilenberg [2]). But, since we are dealing here with a very simple situation, we shall give a direct proof. Since $N N e^{\prime}=0$, the left $A$-module $N e^{\prime}$ is semi-simple and thus $N e^{\prime} \approx \Sigma \Gamma e_{\alpha}$ where the sum is direct and $\left\{e_{\alpha}\right\}$ is an indexed family of primitive idempotents in $\Gamma$. Since $e N e^{\prime} \neq 0$ we have $e \Gamma e_{\alpha} \neq 0$ for at least one index $\alpha$. Thus $e_{\alpha} \approx e$ (meaning $\Gamma e_{\alpha} \approx \Gamma e$ ) and $N e^{\prime}$ has a direct factor isomorphic with $\Gamma e$. Thus

$$
1 . \operatorname{dim}_{\triangle} \Gamma e \leqq 1 \cdot \operatorname{dim}_{\Lambda} N e^{\prime}
$$

Next consider the exact sequence $0 \rightarrow N e \rightarrow \Lambda e \rightarrow \Gamma e \rightarrow 0$. If $\Gamma e$ is not $A$-projective, then

$$
1 . \operatorname{dim}_{\Delta} \Gamma e=1+1 . \operatorname{dim}_{\Lambda} N e \gtrsim 1
$$

which implies the desired result. If $\Gamma e$ is $A$-projective, then the exact sequence splits and we have a direct sum $A e=N e+l$ where $\mathfrak{l}$ is a left ideal of $A$. Multiplying by $N$ we find $N e=N^{2} e+N l=N I \subset$ r. Thus $N e=0$ contrary to hypothesis.

A sequence $\left(e_{0}, \ldots, e_{n}\right)$ of primitive idempotents in $\Gamma$ is called connected if $e_{i-1} N e_{i} \neq 0$ for $i=1, \ldots, n$. The number $n$ is called the length of the connected sequence. It is clear that if in a connected sequence an idempotent is replaced by an isomorphic one, the sequence remains connected.

Proposition 2. A connected sequence of length $n$ exists if and only if $\operatorname{gl} \operatorname{dim} \Lambda \gg n$.

Proof. We may assume $n \gtrsim 1$. The condition $\operatorname{gl} \operatorname{dim} A \geqq n$ is equivalent to 
$1 \cdot \operatorname{dim}_{\Delta} n \geqslant n-1$. Let $\left(e_{0}, \ldots, e_{n}\right)$ be a connected sequence. Then, by Lemma 1 ,

$$
0 \leqslant 1 \cdot \operatorname{dim}_{\Lambda} N e_{i}<1 \cdot \operatorname{dim}_{\Lambda} N e_{i+1} \quad \text { for } i=1, \ldots, n-1 \text {. }
$$

Thus $1 . \operatorname{dim}_{\Lambda} N e \gtrsim n-1$, whence $1 . \operatorname{dim}_{\Lambda} N \geqslant n-1$.

Suppose conversely $1 \cdot \operatorname{dim}_{\Lambda} N \geqslant n-1$. Since $N$ is the direct sum of modules of form $N e$, where $e$ is a primitive idempotent in $\Gamma$, there exists a primitive idempotent $e_{n}$ in $\Gamma$ such that $1 \cdot \operatorname{dim}_{\wedge} N e_{n} \geqslant n-1$. Since $N N e_{n}=0$, the $\Lambda$-module $N e_{n}$ is semi-simple and is therefore the direct sum of modules $\Gamma e$. Thus there exists a primitive idempotent $e_{n-1}$ in $\Gamma$ such that

(i) $\Gamma e_{n-1}$ is isomorphic with a direct suumand of $N e_{n}$,

(ii) $1 . \operatorname{dim}_{\Lambda} \Gamma e_{n-1} \geq n-1$.

Since $e_{n-1} \Gamma e_{n-1} \neq 0$ we have $e_{n-1} N e \neq 0$. Further, from the exact sequence $0 \rightarrow$ $N e_{n-1} \rightarrow \Lambda e_{n-1} \rightarrow \Gamma e_{n-1} \rightarrow 0$ we deduce that $1 \cdot \operatorname{dim}_{\Lambda} N e_{n-1} \geqslant n-2$. Continuing in this fashion we obtain a connected sequence $\left(e_{1}, \ldots, e_{n}\right)$ such that $1 . \operatorname{dim}_{\Delta} N e_{i}$ 르 $i-1$. In particular, $1 . \operatorname{dim}_{\Lambda} N e_{1} \geqslant 0$ i.e. $N e_{1} \neq 0$. There exists therefore a primitive idempotent $e_{0}$ in $\Gamma$ such that $e_{0} N e_{1} \neq 0$. Thus $\left(e_{0}, \ldots, e_{n}\right)$ is a connected sequence of length $n$ as desired.

CoROllary 3. Let $A$ be a semi-primary ring with radical $N$ such that $N^{2}=$ 0 . Let $l$ be the number of simple components of the semi-simple ring $\Gamma=\Lambda / N$. Then

$$
\text { gl. } \operatorname{dim} \Lambda<l \quad \text { or }=\infty \text {. }
$$

Proof. Assume gl.dim $\Lambda \geqslant l$. Then there exists a connected sequence $\left(e_{0}, \ldots, e_{l}\right)$ of primitive idempotents in $I$. At least two of these idempotents must be isomorphic and therefore there exists a connected sequence $\left(e_{0}^{\prime}, \ldots, e_{n}^{\prime}\right)$ with $e_{0}^{\prime}=e_{n}^{\prime}$. This implies the existence of connected sequences of any length. Thus gl. $\operatorname{dim} A=\infty$.

\section{§. The "maximal" ring $\Omega$}

Let $\Gamma$ be a semi-simple ring and $A$ a two-sided $\Gamma$-module. Define $A^{(0)}=\Gamma$, $A^{(n+1)}=A^{(n)} \otimes_{\Gamma} A$. Then define the (graded) ring

$$
\Omega=\sum_{i=0}^{\infty} A^{(i)} \quad \text { (restriced direct sum) }
$$

with multiplication defined by the obvious mapping $A^{(p)} \times A^{(q)} \rightarrow A^{(p+q)}$. Set 
$M=\sum_{i=1}^{\infty} A^{(i)}$. Then

$$
\begin{aligned}
& \Omega=\Gamma+M=\Gamma+A+M^{2}, \\
& M^{k}=\sum_{i=0}^{\infty} A^{(k+i)} .
\end{aligned}
$$

The ring $\Sigma^{\prime}=\Omega / M^{2}$ may be identified with the split extension $\Gamma+A$ (in which $A^{2}=0$ ). Clearly

$$
M=\Omega \otimes_{\Gamma^{\prime}} A .
$$

Since $A$ is projective as a left $\Gamma$-module, it follows that $M$ is projective as a left $\Omega$-module.

Proposition 4. The following conditions are equivalent:

$$
\text { gl. } \operatorname{dim} \Sigma=n \text {, }
$$

$$
A^{(n+1)}=0, A^{(n)} \neq 0 \text {. }
$$

If these conditions hold then $\Omega$ is a hereditary (i.e. gl.dim $\Omega \leqq 1$ ) semiprimary ring with radical $M$ such that $M^{n+1}=0, M^{n} \neq 0$.

Proof. Assume $A^{(n)} \neq 0$. Then there exist elements $a_{1}, \ldots, a_{n} \in A$ and primitive idempotents $e_{1}, f_{1}, \ldots, e_{n}, f_{n} \in \Gamma$ such that

$$
e_{1} a_{1} f_{1} \otimes \ldots \otimes e_{n} a_{n} f_{n} \neq 0
$$

in $A^{(n)}$. Since $e_{i} a_{i} f_{i} \otimes e_{i+1} a_{i+1} f_{i+1}=e_{i} a_{i} \otimes f_{i} e_{i+1} a_{i+1} f_{i+1}$ it follows that $f_{i} e_{i+1} \neq 0$ for $i=1, \ldots, n-1$. Thus $f_{i} \approx e_{i+1}$ for $i=1, \ldots, n-1$ and therefore $\left(e_{1}, f_{1}, f_{2}\right.$, $\left.\ldots, f_{n}\right)$ is a connected sequence of idempotents in $\Gamma$, in the sense of the preceding section (with $\Lambda$ replaced by $\Sigma$ ). Thus, by Proposition 2, gl. $\operatorname{dim} \Sigma \geqslant n$.

Now assume $A^{(n+1)}=0$. Then $\Omega$ is semi-primary with radical $M$ and $M^{n+1}=0$. Since $M$ is projective as a left $\Omega$-module it follows that $\operatorname{gl} \operatorname{dim} \Omega \leqq 1$, i.e. $\Omega$ is hereditary. By Corollary 11 of [3] we have $\mathrm{gl} \cdot \operatorname{dim} \Sigma=\operatorname{gl} \cdot \operatorname{dim}\left(\Omega / M^{2}\right)$ $\leqq n$. This concludes the proof.

\section{§3. Ring in split form}

Let $A$ be a semi-primary ring with radical $N$. A splitting for $\Lambda$ is a direct sum decomposition

$$
A=\Gamma+A+N^{2}
$$


such that

$$
\Gamma \Gamma \subset \Gamma, \Gamma A \subset A, A \Gamma \subset A, A+N^{2}=N .
$$

We have $1 \in \Gamma$. Indeed let $1=\gamma+(1-\gamma)$ with $\gamma \in \Gamma, 1-\gamma \in N$. Then $\gamma=1 \gamma=$ $\gamma^{2}+(1-\gamma) \gamma$ with $\gamma^{2} \in \Gamma$ and $(1-\gamma) \gamma \in N$. Thus $(1-\gamma) \gamma=0$. Consequently $(1-\gamma)^{2}=1-\gamma$. Since $1-\gamma \in N$ it follows that $1-\gamma=0$ i.e. $1=\gamma \in \Gamma$. Thus $\Gamma$ is a subring of $\Lambda$ which may be identified with the semi-simple ring $\Lambda / N$, and $A$ is a two-sided $I$-module which may be identified with $N / N^{2}$. The ring $A / N^{2}$ may be identified with the split extension $\Sigma=T+A$.

TheOREM 5. Let $A$ be a semi-primary ring with radical $N$ such that $A$ admits a splitting and

$$
\text { gl. } \operatorname{dim}\left(A / N^{2}\right)=n<\infty .
$$

Then there exist a hereditary semi-primary ring $\Omega$ with radical $M$ and a ring epimorphism $\varphi: \Omega \rightarrow \Lambda$ such that $\varphi^{-1}\left(N^{2}\right)=M^{2}$ i.e. $\varphi$ induces an isomorphism

$$
\Omega / M^{2} \approx \Lambda / N^{2}
$$

The pair $(\Omega, \varphi)$ is determined uniquely up to an isomorphism. Moreover, the ring $\Omega$ admits a splitting, $M^{n+1}=0$, and $N^{n+1}=0$.

Corollary 6. With 4 as in Theorem 5

$$
\text { gl. } \operatorname{dim}(\Lambda / a)<\infty
$$

for every two-sided ideal $a$ in $A$. If $a \subset N^{2}$ then

$$
\operatorname{gl} \operatorname{dim}(\Lambda / a) \leqq n .
$$

Inparticular,

$$
\text { gl. } \operatorname{dim} A \leqq n .
$$

If $l$ is the number of simple components of $\Gamma=\Lambda / N$ then $n<l$.

Proof. Let $A=\Gamma+A+N^{2}$ be a splitting for $\Lambda$. Let $\Omega$ be the ring constructed in $\S 2$ using the ring $\Gamma$ and the two-sided $\Gamma$-module $A$. Since $\Sigma=\Lambda / N^{2}$ we have gl. $\operatorname{dim} \Sigma=n<\infty$. Thus, by Proposition $4, \Omega$ is a semi-primary ring with radical $M$ and $M^{n+1}=0$. Define the ring homomorphism $\varphi: \Omega \rightarrow \Lambda$ by setting $\varphi(\gamma)=\gamma$ for $\gamma \in \Gamma$ and $\varphi\left(a_{1} \otimes \ldots \otimes a_{k}\right)=a_{1} \ldots a_{k}$ for $a_{1} \otimes \ldots \otimes a_{k}$ $\in A^{(k)}, k>0$. We have $A \subset \varphi(M) \subset N$. It follows that $N=\varphi(M)+N^{2}$. There- 
fore $N=\varphi(M)$ and $\varphi$ is an epimorphism. Clearly $\Omega$ admits a splitting $\Omega=\Gamma+$ $A+M^{2}$, and $\varphi^{-1}\left(N^{2}\right)=M^{2}$.

Let $\Omega^{\prime}$ be another hereditary semi-primary ring with radical $M^{\prime}$ and let $\varphi^{\prime}: \Omega^{\prime} \rightarrow \Lambda$ be a ring epimorphism such that $\varphi^{\prime-1}\left(N^{2}\right)=M^{\prime 2}$. There results for $\Omega^{\prime}$ a splitting $\Omega^{\prime}=\varphi^{\prime-1}(\Gamma)+\varphi^{\prime-1}(A)+M^{\prime 2}$. If we identify $\varphi^{\prime-1}(\Gamma)$ with $\Gamma$ and $\varphi^{\prime-1}(A)$ with $A$ using the mapping $\varphi^{\prime}$ we obtain a splitting $\Omega^{\prime}=\Gamma+A+M^{\prime}$ and $\varphi^{\prime}$ is the identity on $\Gamma+A$. If we replace $A$ by $\Omega^{\prime}$ in the construction above we obtain an epimorphism $\psi: \Omega \rightarrow \Omega^{\prime}$ such that $\phi^{-1}\left(M^{\prime 2}\right)=M^{2}$. Since the ring homomorphisms $\varphi, \varphi^{\prime} \psi: \Omega \rightarrow \Lambda$ coincide on $\Gamma+A$, it follows that $\varphi=\varphi^{\prime} \psi$. There remains to be shown that $\psi$ is an isomorphism. Let $a$ be the kernel of $\psi$. Then $\Omega / a \approx \Omega^{\prime}$ and $a \subset M^{2}$. It follows then from Theorem I of [4] (or [3], Proposition 10 and Remark there) that $a=0$. Since $M^{n+1}=0$ and $N=\varphi(M)$ we have $N^{n+1}=0$. This concludes the proof of the theorem.

The last statement of the corollary follows from Corollary 3 applied to the ring $\Sigma^{\prime}=\Gamma+A=\Lambda / N^{2}$.

Let $a$ be any two-sided ideal in $\Lambda$ and let $b=\varphi^{-1}(a)$. Then $\Lambda / a \approx \Omega / b$ so that by [3], Theorem $8, \operatorname{gl} \cdot \operatorname{dim}(\Lambda / a)<\infty$.

If $a \subset N^{2}$ then $b \subset M^{2}$ and the conclusion that $\operatorname{gl} \operatorname{dim}(\Lambda / a) \leqq n$ is then a consequence of

PROPOSITION 7. Let $\Omega$ be a hereditary semi-primary ring with radical $M$ such that $M^{n+1}=0$. For any two-sided ideal $b \subset M^{2}$

$$
\operatorname{gl} \operatorname{dim}(\Omega / b) \leqq n .
$$

Proof. Assume $n$ even, $n=2 i$. We may assume $i>0$ since if $i=0$ then $M=0, b=0$ and $\Omega=\Omega / b$ is semi-simple. Since $b \subset M^{2}$ and $M^{2 i+1}=0$ it follows that $b^{i} M=b^{i+1}=0$. Thus [3] Proposition 9, condition (iii') implies $\operatorname{gl}$. $\operatorname{dim}(\Omega / b$ ) $\leqq n$.

Let $n$ be odd, $n=2 i+1$. We may assume $i>0$ since if $i=0$ then $n=1$, $M^{2}=0, b=0$ and $\mathrm{gl} \cdot \operatorname{dim}(\Omega / b)=\mathrm{gl} \cdot \operatorname{dim} \Omega \leqq 1$ by hypothesis. Since $b \subset M^{2}$ and $M^{2 i+2}=0$ it follows that $b^{i+1}=0$. Thus [3] Proposition 9, condition (iii) implies $\operatorname{gl.dim}(\Omega / b) \leqq n$.

Next we consider a semi-primary ring $A$, with radical $N$ and admitting a splitting $\Lambda=\Gamma+A+N^{2}$, which satisfies

$$
\operatorname{gl} \cdot \operatorname{dim}\left(\Lambda / N^{2}\right)=\infty
$$


contrary to Theorem 5. Again construct $\Omega$ as in $\S 2$ using the ring $A$ and the two-sided $A$-module $A$, and let $M$ have the same significance as before. Let $N^{h}=0$. Then $\Lambda$ is a homomorphic image of $\Omega / M^{m}$ for every $m \geqslant h$. We want to show

PROPOSITION 8. (Under our assumption gl. $\operatorname{dim}\left(A / N^{2}\right)=\infty$ ) the semi-primary ring $\Omega / M^{m}$ has gl.dimension $\infty$ for infinitely many $m$.

Proof. By our assumption gl. $\operatorname{dim}\left(\Lambda / N^{2}\right)=\infty$, there exists a connected sequence $\left(e_{0}, e_{1}, \ldots, e_{k-1}, e_{0}\right)(k \neq 0)$ of primitive idempotents in $\Gamma$, with respect to $\Lambda / N^{2}$, whose first and last terms coincide. We contend that gl.dim $\left(\Omega / M^{2 k}\right)=\infty$. To see this, consider the left $\left(\Omega / M^{2 k}\right)$-module $\left(\Omega / M^{k}\right) \boldsymbol{e}_{0}$. We have the exact sequence

$$
0 \longrightarrow\left(M^{k} / M^{2 k}\right) e_{0} \longrightarrow\left(\Omega / M^{2 k}\right) e_{0} \longrightarrow\left(\Omega / M^{k}\right) e_{0} \longrightarrow 0 .
$$

Let $1=e_{0}+\Sigma f_{\nu}$ be a decomposition of 1 into mutually orthogonal primitive idempotents in $\Gamma$. We have $M^{k}=\Omega \otimes_{\Gamma} A^{(k)}=\Omega \otimes_{\Gamma} e_{0} A^{(k)}+\Sigma \Omega \otimes_{\Gamma} f_{\nu} A^{(k)}$ (direct). Hence $M^{k} e_{0}=\Omega \otimes_{\Gamma} e_{0} A^{(k)} e_{0}+\Sigma \Omega \otimes_{\Gamma} f_{\nu} A^{(k)} e_{0}$ (direct). As $M^{2 k}=M^{k} \otimes_{\Gamma} A^{(k)}$, we have similarly $M^{2 k} e_{0}=M^{k} \otimes_{\Gamma} e_{0} A^{(k)} e_{0}+\Sigma M^{k} \otimes_{\Gamma} f_{\nu} A^{(k)} e_{0}$ (direct). Then we obtain readily

$$
\left(M^{k} / M^{2 k}\right) e_{0} \approx\left(\Omega / M^{k}\right) \otimes_{\Gamma} e_{0} A^{(k)} e_{0}+\Sigma\left(\Omega / M^{k}\right) \otimes_{\Gamma} f_{\nu} A^{(k)} e_{0} \quad \text { (direct). }
$$

Since $\left(e_{0}, e_{1}, \ldots, e_{k-1}, e_{0}\right)$ is connected, we have here $e_{0} A^{(k)} e_{0} \neq 0$. On taking a left $e_{0} \Gamma e_{0}$-basis of $e_{0} A^{(k)} e_{0}$ we then obtain an isomorphism

$$
\left(M^{k} / M^{2 k}\right) e_{0} \approx\left(\Omega / M^{k}\right) e_{0}+W \quad \text { (direct) }
$$

where $W$ is a left $\left(\Omega / M^{2 k}\right)$-module whose structure does not concern us. Thus we have the exact sequence

$$
0 \longrightarrow\left(\Omega / M^{k}\right) e_{0}+W \longrightarrow\left(\Omega / M^{2 k}\right) e_{0} \longrightarrow\left(\Omega / M^{k}\right) e_{0} \longrightarrow 0 .
$$

Now, suppose $r=1 . \operatorname{dim}_{\Omega / M^{2 k}}\left(\Omega / M^{k}\right) \boldsymbol{e}_{0}<\infty$ and let

$$
0 \longrightarrow X_{r} \longrightarrow \ldots \rightarrow X_{1} \longrightarrow X_{0} \rightarrow\left(\Omega / M^{k}\right) e_{0} \rightarrow 0
$$

be a shortest $\left(\Omega / M^{2 k}\right)$-projective resolution of $\left(\Omega / M^{k}\right) e_{0}$; we have $r>0$ since $\left(\Omega / M^{k}\right) e_{0}$ is not $\left(\Omega / M^{2 k}\right)$-projective. We have then an exact sequence

$$
0 \longrightarrow X_{r}+Y_{r} \longrightarrow \ldots \rightarrow X_{0}+Y_{0} \longrightarrow\left(\Omega / M^{2 k}\right) e_{0} \longrightarrow\left(\Omega / M^{k}\right) e_{0} \longrightarrow 0,
$$

where sums are all direct and where $0 \rightarrow Y_{r} \rightarrow \ldots \rightarrow Y_{0} \rightarrow W \rightarrow 0$ is an 
exact sequence such that all $Y_{\mu}$ except $Y_{r}$, perhaps, are $\left(\Omega / M^{2 k}\right)$-projective. Since $1 . \operatorname{dim}_{\Omega / M^{2 k}}\left(\Omega / M^{k}\right) e_{0}=r$, then necessarily the image of $X_{r}+Y_{r}$ in $X_{r-1}+Y_{r-1}$ is a direct summand. Hence the image of $X_{r}$ in $X_{r-1}$ is a direct summand. This in turn implies that $\left(\Omega / M^{k}\right) e_{0}$ has a projective resolution, with respect to $\Omega / M^{2 k}$, of length $r-1$, contradicting the above assumption. Hence $1 . \operatorname{dim} \Omega_{\Omega / M^{2 k}}\left(\Omega / M^{k}\right) \boldsymbol{e}_{0}$ $=\infty$ and $\operatorname{gl} \cdot \operatorname{dim}\left(\Omega / M^{2 k}\right)=\infty$.

Here we may assume that $k$ is arbitrarily large, since otherwise we have simply to repeat the given connected sequence of idempotents sufficiently many times. So this proves our proposition.

\section{\$. Algebras}

Let $\Lambda$ be a semi-primary algebra over a field $K$, let $N$ be the radical of $\Lambda$ and let $\Gamma=\Lambda / N$. Assume $\operatorname{dim} \Gamma=0$, or equivalently that $\Gamma \otimes_{K} \Gamma^{*}$ is semi-simple. Then (Rosenberg-Zelinsky [8]) necessarily $(\Gamma: K)<\infty$ and $\Gamma$ is separable. It follows readily that $\Lambda$ admits a splitting $\Lambda=\Gamma+A+N^{2}, A \approx N / N^{2}$. It is further known (Eilenberg [1]) that $\operatorname{dim} \Lambda=\operatorname{gl} . \operatorname{dim} \Lambda$. Similarly if $a$ is any twosided ideal in $\Lambda$ then $\operatorname{dim}(\Lambda / a)=\operatorname{gl} \cdot \operatorname{dim}(\Lambda / a)$.

The same comments apply to the algebra $\Omega$ constructed in $\S 2$, provided $M$ is nilpotent. The results of $\S 3$ may now be restated with "dim" replacing "gl.dim".

If we assume that $(A: K)<\infty$ then clearly $A$ is semi-primary and the assumption $\operatorname{dim} \Gamma=0$ (i.e. the separability of $\Gamma$ ) follows automatically from $\operatorname{dim}\left(\Lambda / N^{2}\right)<\infty$ (Ikeda-Nagao-Nakayama [7], Eilenberg [1]). It is further clear that in the splitting $\Lambda=\Gamma+A+N^{2}$ of $\Lambda$ we have $(A: K)<\infty$. Since $\Omega=\Gamma+M$ we deduce that $(\Omega: K)<\infty$. Thus we have

Theorem 9. Let $\Lambda$ be an algebra over a field $K$ with $(\Lambda: K)<\infty$. Let $N$ be the radical of $\Lambda$. Suppose

$$
\operatorname{dim}\left(\Lambda / N^{2}\right)=n<\infty .
$$

Then there exist an algebra $\Omega$ over $K$ with radical $M$ and an algebra epimorphism $\varphi: \Omega \longrightarrow \Lambda$ such that $(\Omega: K)<\infty, \varphi^{-1}\left(N^{2}\right)=M^{2}$ and

$$
\operatorname{dim} \Omega \leqq 1 .
$$

The pair $(\Omega, \varphi)$ is determined uniquely up to an isomorphism. $M^{n+1}=0$ and $N^{n+1}=0$. If $a$ is a two-sided ideal of $A$ then $\operatorname{dim}(\Lambda / a)<\infty$, and indeed $\leqq n$ if 
$a \subset N^{2}$. If $l$ is the number of simple components in $\Gamma=1 / N$ then $n<l$.

We close our note with a remark on Cartan matrices. Starting again with a semi-primary ring $\Lambda$, with radical $N$, let $e_{1}, \ldots, e_{l}$ be a maximal set of nonisomorphic primitive idempotents in $A$. For each pair $(i, j)$ of indices $1,2, \ldots$, $l$ we choose a non-negative real number $\beta(i, j)$ so that

$$
\beta(i, j)=0 \text { or }>0 \text { according as } e_{i} N e_{j}=0 \text { or } \neq 0,
$$

and otherwise arbitrarily. Let us call the matrix $C(\Lambda)=I+(\beta(i, j))$ a generalized Cartan matrix of $A$, where $I$ is the identity matrix of degree $l$.

Proposition 10. The matrix $(C(A)-I)^{n+1}=(\beta(i, j))^{n+1}$ vanishes if and only if $\operatorname{gl} \operatorname{dim}\left(\Lambda / N^{2}\right) \leqq n$.

Proof. Since the entries $\beta(i, j)$ of $C(\Lambda)-I$ are all non-negative, that $(C(\Lambda)-I)^{n+1} \neq 0$ is equivalent to the existence of $n+1$ pairs $\left(i_{0}, j_{0}\right), \ldots$, $\left(i_{n}, j_{n}\right)$ such that

$$
j_{\nu}=i_{\nu+1}(\nu=0, \ldots, n-1), \beta\left(i_{\nu}, j_{\nu}\right) \neq 0 \quad(\nu=0, \ldots, n) .
$$

By the definition of $\beta(i, j)$, this is equivalent to

$$
j_{\nu}=i_{\nu+1}(\nu=0, \ldots, n-1), e_{i_{\nu}} N e_{j_{\nu}} \neq 0 \quad(\nu=0, \ldots, n) .
$$

Now, if $e N^{t} f \neq 0$ but $e N^{t+1} f=0$, with a pair of primitive idempotents $e, f$ in $A$, take $t-1$ primitive idempotents $g_{1}, \ldots, g_{t-1}$ such that $N g_{1} N g_{2} \ldots N g_{t-1} N f$ $\neq 0$. Since $e N^{t+1} f=0$, it follows that $g_{\mu} N g_{\mu+1} \notin N^{2}$ for $\mu=0, \ldots, t-1$, where we put $g_{0}=e, g_{t}=f$. This observation shows that the existence of $n+1$ pairs $\left(i_{\nu}, j_{\nu}\right)$ satisfying (ii) is equivalent to the existence of a connected sequence of length at least $n+1$ of primitive idempotents in $\Gamma=\Lambda / N$, with respect to $\Lambda / N^{2}$, in the sense of $\S 1$. This is in turn equivalent to $\operatorname{gl} \cdot \operatorname{dim}\left(\Lambda / N^{2}\right) \geq n+1$ by Proposition 2 .

In case of an algebra $\Lambda$ over a field $K$ with $(\Lambda: K)<\infty$, the ordinary Cartan matrix of $A$ is clearly a generalized Cartan matrix in the above sense.

\section{References}

[1] S. Eilenberg, Algebras of cohomologically finite dimension, Comment. Math. Helv. 28 (1954), 310-319.

[2] S. Eilenberg, Homological dimension and syzygies, to appear in Ann. Math.

[3] S, Eilenberg, H. Nagao and T. Nakayama, On the dimension of modules and algebras, 
IV, Nagoya Math. J. 10 (1956), 87-95.

[4] S. Eilenberg and T. Nakayama, On the dimension of modules and algebras, V, Nagoya Math. J. 11 (1957), 9-12.

[5] G. Hochschild, On the structure of algebras with nonzero radical, Bull. Amer. Math. Soc. 53 (1947), 369-377.

[6] G. Hochschild, Note on maximal algebras, Proc. Amer. Math. Soc. 1(1950), 11-14.

[7] M. Ikeda, H. Nagao and T. Nakayama, Algebras with vanishing $n$-cohomology grops, Nagoya Math. J. 7 (1954), 115-131.

[8] J. P. Jans, On segregated rings and algebras, Nagoya Math. J. 11 (1957).

[9] A. Rosenberg and D. Zelinsky, Cohomology of infinite algebras, Trans. Amer. Math. Soc. 82 (1956), 85-98.

Yale University,

Nagoya University and

The Institute for Advanced Study 